\title{
Modelling the polarised emission from black holes on event horizon-scales
}

\author{
Ziri Younsi ${ }^{1,2}$, Oliver Porth ${ }^{1}$, Yosuke Mizuno ${ }^{1}$, Christian M. Fromm ${ }^{1,3}$ \\ and Hector Olivares ${ }^{1}$ \\ ${ }^{1}$ Institut für Theoretische Physik, Max-von-Laue-Straße 1, \\ D-60438 Frankfurt am Main, Germany \\ email: younsi@itp.uni-frankfurt.de \\ ${ }^{2}$ Mullard Space Science Laboratory, University College London, \\ Holmbury St. Mary, Dorking, Surrey RH5 6NT, UK \\ email: z.younsi@ucl.ac.uk \\ ${ }^{3}$ Max-Planck-Institut für Radioastronomie, Auf dem Hügel 69, D-53121 Bonn, Germany
}

\begin{abstract}
Upcoming VLBI observations will resolve nearby supermassive black holes, most notably Sagittarius A* and M87, on event horizon-scales. Recent observations of Sagittarius A* with the Event Horizon Telescope have revealed horizon-scale structure. Accordingly, the detection and measurement of the back hole "shadow" is expected to enable the existence of astrophysical black holes to be verified directly. Although the theoretical description of the shadow is straightforward, its observational appearance is largely determined by the properties of the surrounding accretion flow, which is highly turbulent. We introduce a new polarised generalrelativistic radiative transfer code, BHOSS, which accurately solves the equations of polarised radiative transfer in arbitrary strong-gravity environments, providing physically-realistic images of astrophysical black holes on event horizon-scales, as well as also providing insight into the fundamental properties and nature of the surrounding accretion flow environment.
\end{abstract}

Keywords. gravitation, methods: numerical, radiative transfer, relativity, polarisation

\section{Introduction}

It is widely believed that all galaxies host a supermassive black hole (SMBH) at their center. Advances in very-long-baseline-interferometry (VLBI) have enabled the Event Horizon Telescope Collaboration (EHTC) to image (with event horizon-scale resolution) the nearby SMBHs Sagittarius A* (Sgr A*) and M87, with the first observational results expected soon (e.g., Doeleman et al. 2008, Goddi et al. 2017). When observing an astrophysical black hole, theorists anticipate seeing a "shadow", the silhouette of the unstable photon region surrounding the event horizon (e.g., Cunningham \& Bardeen 1973, Grenzebach et al. 2014, Younsi et al. 2016). Gravity near the event horizon is so strong that spacetime is curved and light rays no longer travel along straight lines. Moreover, the surrounding environment is hot, magnetised and turbulent, serving to obscure and modify the shadow's observed properties. As such, both the equations of motion for light rays and the radiative transfer equation must be solved in full general relativity.

We present results from our new general-relativistic radiative transfer (GRRT) code, BHOSS (Younsi et al. 2018, in prep.), which solves the equations of polarised radiative transfer for arbitrary spacetime metrics (e.g., Younsi et al. 2016, Mizuno et al. 2018), and is fully coupled to many three-dimensional general-relativistic magnetohydrodynamical (GRMHD) codes, including BHAC (Porth et al. 2017) and HARM (Gammie et al. 2003). 

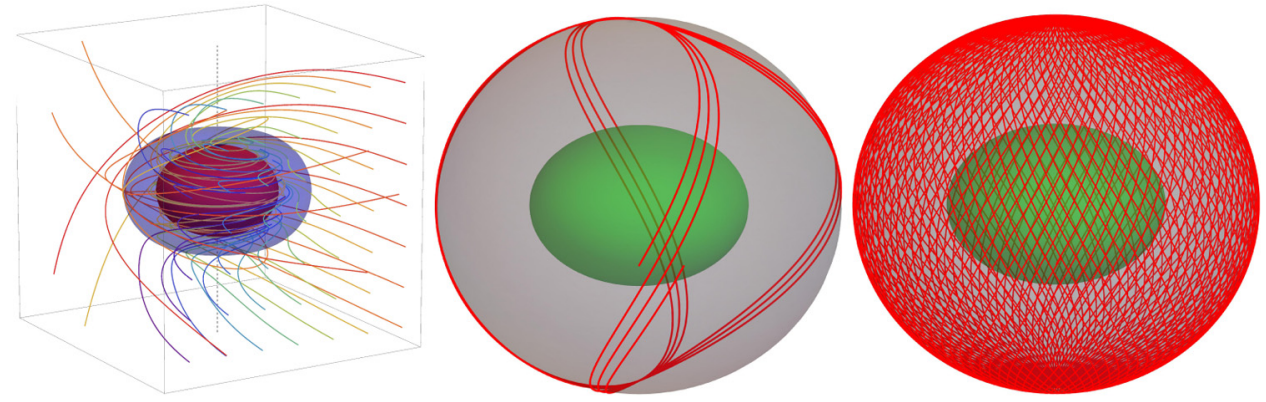

Figure 1. Photon geodesics around an extremal Kerr black hole. Left panel: $7 \times 7$ pixel observer grid of rays with event horizon in red and ergo-region in blue. Middle panel: $6 \pi$ longitudinal oscillations of a spherical photon orbit (event horizon in green). Right panel: $100 \pi$ longitudinal oscillations of the spherical photon orbit. The orbit is stable for long integration times.

\section{General-relativistic ray-tracing and radiative transfer}

Ray-tracing. In order to construct an image of a black hole, the paths of light rays (photons) comprising an image are ray-traced by solving the geodesic equations of motion:

$$
\begin{aligned}
& \frac{\mathrm{d} x^{\alpha}}{\mathrm{d} \lambda}=k^{\alpha}, \\
& \frac{\mathrm{d} k^{\alpha}}{\mathrm{d} \lambda}=-\Gamma_{\mu \nu}^{\alpha} k^{\mu} k^{\nu},
\end{aligned}
$$

where $x^{\alpha}$ and $k^{\alpha}$ are, respectively, the photon position and 4-momentum, $\Gamma_{\mu \nu}^{\alpha}$ denote the Christoffel symbols, $\lambda$ is the affine parameter and Greek indices range from 0-3 throughout. Appropriate initial conditions may be determined using a pre-defined observer "camera" (see, e.g., Younsi et al. 2016 and left panel of Fig. 1). Tensor index raising and lowering operations are performed with the metric tensor, $g_{\mu \nu}$, e.g., $k_{\mu}=g_{\mu \nu} k^{\nu}$. Geodesics in any spacetime may be calculated by specifying the appropriate $g_{\mu \nu}$.

Numerical errors in geodesic integration grow over time, and are minimised in BHOSS by using several different high-order integration schemes. A robust test of stability is the long-term integration of spherical photon orbits (e.g., Teo 2003, Chan et al. 2017), which are notoriously challenging to maintain for long times but readily maintained in BHOSS (see middle and right panels of Fig. 1).

Polarised radiative transfer. Along each ray (image pixel) the intensity and polarisation properties are calculated from the equations of polarised radiative transfer. The observer's polarisation basis can be represented by parallel and perpendicular 4-vectors of this basis, $f_{(\|)}^{\alpha}$ and $f_{(\perp)}^{\alpha}$, which are orthogonal both to each other and to $k^{\alpha}$, and solved as:

$$
\frac{\mathrm{d} f^{\alpha}}{\mathrm{d} \lambda}=-\Gamma_{\mu \nu}^{\alpha} k^{\mu} f^{\nu}
$$

Defining the Lorentz-invariant Stokes vector as $\mathcal{S}:=(\mathcal{I}, \mathcal{Q}, \mathcal{U}, \mathcal{V})^{\mathrm{T}}$, where $\mathcal{S} \equiv \mathbf{S} / \nu^{3}$ and $\mathbf{S}:=(I, Q, U, V)^{\mathrm{T}}$, the full polarised GRRT equation may be written as:

$$
\frac{\mathrm{d}}{\mathrm{d} \lambda}\left(\begin{array}{c}
\mathcal{I} \\
\mathcal{Q} \\
\mathcal{U} \\
\mathcal{V}
\end{array}\right)=-k_{\mu} u^{\mu}\left\{R(\chi)\left(\begin{array}{c}
\bar{\varepsilon}_{I} \\
\bar{\varepsilon}_{Q} \\
0 \\
\bar{\varepsilon}_{V}
\end{array}\right)-\left[R(\chi)\left(\begin{array}{cccc}
\bar{\alpha}_{I} & \bar{\alpha}_{Q} & 0 & \bar{\alpha}_{V} \\
\bar{\alpha}_{Q} & \bar{\alpha}_{I} & \bar{\rho}_{V} & 0 \\
0 & -\bar{\rho}_{V} & \bar{\alpha}_{I} & \bar{\rho}_{Q} \\
\bar{\alpha}_{V} & 0 & -\bar{\rho}_{Q} & \bar{\alpha}_{I}
\end{array}\right) R(-\chi)\right]\left(\begin{array}{c}
\mathcal{I} \\
\mathcal{Q} \\
\mathcal{U} \\
\mathcal{V}
\end{array}\right)\right\}
$$



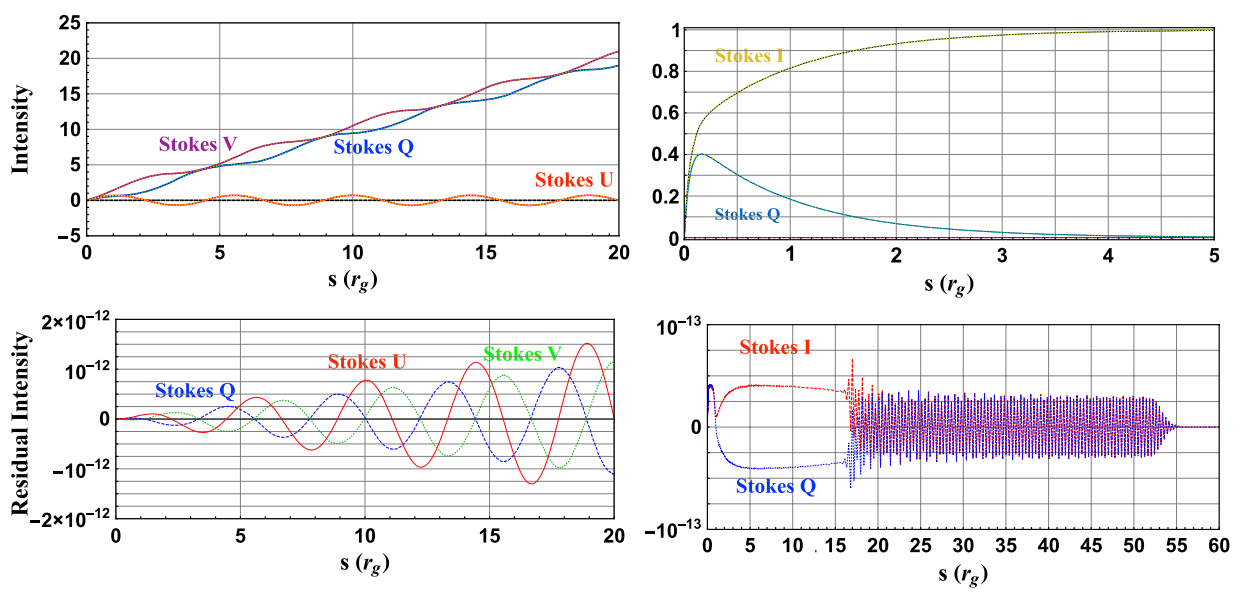

Figure 2. Polarised GRRT tests I. Left column: pure polarised emission in Stokes $(Q, U, V)$, $I=0$ (top panel) and residuals compared to analytic solution (bottom panel). Right column: pure emission and absorption in Stokes $(I, Q), U=V=0$ (top panel) and residuals compared to analytic solution (bottom panel). Analytic solutions are plotted as dashed lines in upper panels.

where $\bar{\varepsilon}_{i}$ are the invariant emissivities, $\bar{\alpha}_{i}$ the invariant absoprtivities and $\bar{\rho}_{i}$ are the invariant Faraday rotation and conversion coefficients. Invariant (barred) quantities are related to their standard (fluid rest frame) counterparts as $\bar{\varepsilon}_{i}=\varepsilon_{i} / \nu^{2}, \bar{\alpha}_{i}=\nu \alpha_{i}$ and $\bar{\rho}_{i}=$ $\nu \rho_{i}$, where $\nu$ is the frequency (see Younsi et al. 2012). The 4-velocity of the fluid, e.g., an accretion flow around a black hole, is denoted by $u^{\mu}$ and $R(\chi)$ is a rotation matrix where $\chi \in[-\pi, \pi]$ is the angle between the polarisation basis and the plasma magnetic field, also termed the electric vector position angle (EVPA). Note that $\varepsilon_{U}=\alpha_{U}=\rho_{U}=0$ is fixed by rotating to a frame aligning the magnetic field with $U$. Equation (2.4) is integrated along each photon geodesic, returning the Stokes parameters, $\mathbf{S}$.

\section{Preliminary tests and future work}

We present preliminary tests of the above polarised GRRT framework in BHOSS. Several polarised GRRT codes exist in the literature (Broderick \& Blandford 2003a, 2003b, Schnittman \& Krolik 2010, Shcherbakov \& Huang 2011, Dexter 2016, Mościbrodzka \& Gammie 2018, Pihajoki et al. 2018) which contain several standard tests. Figure 2 shows two ray transport tests (see Dexter 2016): (i) transport of pure polarised emission with Faraday rotation and conversion, where $\epsilon_{Q}=\epsilon_{U}=\epsilon_{V}=\rho_{Q}=\rho_{V}=1, \rho_{U}=0$, and (ii) pure emission and absorption in Stokes I and Q, with $\varepsilon_{I}=\alpha_{I}=10, \varepsilon_{Q}=\alpha_{Q}=9$. The numerical integration performs well and, as expected, Stokes $I$ is zero to machine precision in test (i) and Stokes $U$ and $V$ are similarly zero in test (ii).

In Fig. 3 we present more detailed polarised GRRT imaging tests. The left panel shows the EVPA map of a thin Keplerian accretion disk around a Schwarzschild black hole, and is in good agreement with that of Fig. 3 in Dovčiak et al. 2008. The right panel presents the image of a Novikov-Thorne accretion disk with an electron scattering atmosphere (see Schnittman \& Krolik 2010 for further details). The obtained image is in good qualitative agreement with previous results obtained by Schnittman \& Krolik 2010 and Dexter 2016.

We have presented a numerical GRRT code capable of integrating the equations of polarised GRRT to high accuracy in arbitrary spacetime geometries, demonstrating excellent agreement with previous results and analytic solutions in the literature. Since BHOSS already performs GRRT in time-dependent optically-thin and optically-thick GRMHD backgrounds, and for arbitrary spacetime geometries, we will next perform polarised 

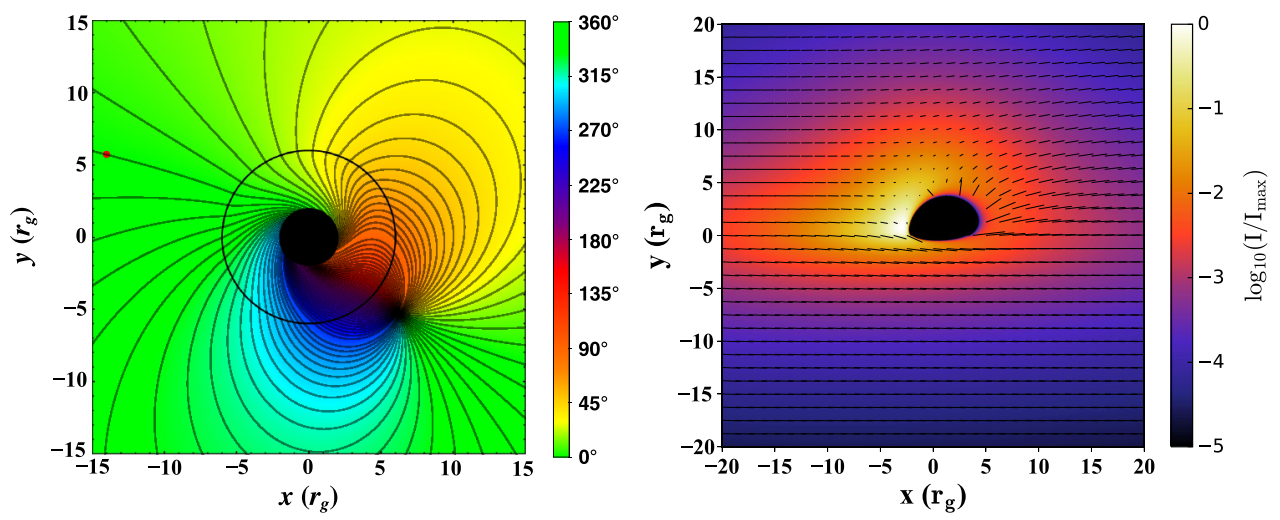

Figure 3. Polarised GRRT tests II. Left panel: EVPA contour map $(\widetilde{\chi} \equiv \chi+\pi)$ of a Keplerian accretion disk around a Schwarzschild $\mathrm{BH}$ as viewed at $30^{\circ}$. The black ring denotes the innermost stable circular orbit (ISCO), wherein the disk material is in free-fall. The red dot corresponds to the $\tilde{\chi}=0^{\circ}$ contour. Right panel: Novikov-Thorne accretion disk with electron scattering atmosphere around an extremal Kerr black hole. Colour denotes the total intensity integrated over the range $0.1-10 \mathrm{keV}, \dot{M}=0.1 M_{\mathrm{Edd}}, M=10 M_{\odot}$ and viewed at $75^{\circ}$. Orientation of black ticks indicates the polarisation direction, and the length of each tick denotes the polarisation degree ( $\sim 5 \%$ at bottom of panel). Significant depolarisation and enhancement can be seen.

GRRT calculations on GRMHD simulation data, determining time-variable polarised emission from accreting black holes and providing meaningful theoretical predictions to be compared with upcoming and future observing campaigns.

\section{Acknowledgements}

We thank Luciano Rezzolla, Hung-Yi Pu and Thomas Bronzwaer for helpful input. Support comes from the ERC Synergy Grant "BlackHoleCam - Imaging the Event Horizon of Black Holes" (Grant 610058). ZY is supported by a Leverhulme Trust Early Career Fellowship. This research has made use of NASA's Astrophysics Data System.

\section{References}

Doeleman, S. S., Weintroub, J., Rogers, A. E. E., Plambeck, R., et al. 2008, Nature, 78-80, 455 Goddi, C., Falcke, H., Kramer, M., et al. 2017, Int. J. Mod. Phys. D, 26, 1730001-239

Cunningham, C. T. and Bardeen, J. M. 1973, Astrophys. J., 237-264, 183

Grenzebach, A., Perlick, V. and Lämmerzahl, C. 2014, Phys. Rev. D, 12, 89

Younsi, Z., Zhidenko, A., Rezzolla, L., Konoplya, R. \& Mizuno, Y. 2016, Phys. Rev. D, 8, 94

Mizuno, Y., Younsi, Z., Fromm, C. M., Porth, O., De Laurentis, M., Olivares, H., Falcke, H., Kramer, M. \& Rezzolla, L. 2018, Nature Astronomy, 2, 585-590

Porth, O., Olivares, H., Mizuno, Y., Younsi, Z., Rezzolla, L. et al. 2017, CA\&C, 1, 4

Gammie, C. F., McKinney, J. C. \& Tóth, G. 2003, Astrophys. J., 458, 589

Teo, E. 2003 Gen. Rel. and Grav., 35, 11

Chan, C.-k., Medeiros, L., Ozel, F. \& Psaltis, D. 2017, ArXiv e-prints:1706.07062

Younsi, Z., Wu, K. and Fuerst, S. V. 2012, Astron. Astrophys., A13, 545

Broderick, A., \& Blandford, R. 2003a, MNRAS, 342, 1280; 2003b, Ap\&SS, 288, 161

Shcherbakov, R. V. \& Huang, L. 2011, MNRAS, 410, 1052

Dexter, J. 2016, MNRAS, 462, 115

Mościbrodzka, M. \& Gammie, C. F. 2018, MNRAS, 475, 43-54

Pihajoki, P., Mannerkoski, M., Nättilä, J. \& Johansson, P. H. 2018, Astrophys. J., 863, 8

Schnittman, J. D. \& Krolik, J. H. 2010, Astrophys. J., 712, 908-924

Dovčiak, M., Muleri, F., Goosmann, R. W., Karas, V. \& Matt, G. 2008, MNRAS, 391, 32-38 